\title{
Long-lasting tuberculous pleurisy
}

\author{
To the Editor:
}

We read with great interest the paper by PeTRUCCioli et al. [1] where the authors describe new prognostic tests and different approaches that can predict the progression from latent to active tuberculosis (TB). We have taken the opportunity to comment on this paper to describe a recent case of recurrent tuberculous infection in a patient with a long-lasting history of tuberculous pleurisy and secondary residual pleural thickening.

At the age of 33 the patient had suffered from a left tuberculous pleurisy treated by anti-TB therapy, including daily intramuscular streptomycin $\left(15 \mathrm{mg} \cdot \mathrm{kg}^{-1}\right.$ for $\left.2 \mathrm{months}\right)$, daily oral rifampin $\left(10 \mathrm{mg} \cdot \mathrm{kg}^{-1}\right.$ for 6 months) and daily oral isoniazid ( $5 \mathrm{mg} \cdot \mathrm{kg}^{-1}$ for 6 months) combined with pleural drainage. At that time, Mycobacterium tuberculosis was isolated from sputum and pleural fluid. At the age of 46, the patient underwent examination for a blunt thoracic trauma and a chest radiograph showed a chronic left pleural effusion likely due to his previous pleurisy (figure 1a); however, no treatment was proposed.

At 65 years of age, the patient (a non-smoking man) presented with chest pain and dyspnoea. A chest radiograph showed an enlargement of the pleural collection (figure $1 \mathrm{~b}$ ) and a computed tomography (CT)-scan of the chest confirmed the presence of a calcifying pleurisy leading to a contralateral mediastinal shift and lung atelectasis (figures $1 \mathrm{c}$ and 1e). Bronchoscopy was negative, the patient was afebrile and blood gas examination showed oxygen tension $\left(\mathrm{PO}_{2}\right)$ of $67 \mathrm{mmHg}$, carbon dioxide tension $\left(\mathrm{PCO}_{2}\right)$ of $46 \mathrm{mmHg}$ and oxygen saturation of $94 \%$. Pulmonary function tests revealed a moderate restrictive pattern. White blood cell count was normal and C-reactive protein level was elevated $\left(152 \mathrm{mg} \cdot \mathrm{L}^{-1}\right.$; range $\left.0-5 \mathrm{mg} \cdot \mathrm{L}^{-1}\right)$, while a Quantiferon-TB test was negative. Considering his previous history and the actual respiratory symptoms, a chest tube was positioned and a brownish purulent effusion $(600 \mathrm{~mL})$ was drained which had an observed lymphocyte percentage of $88 \%$. Its bacteriological examination revealed the presence of Streptococcus pyogenes, while testing for $M$. tuberculosis and other atypical mycobacteria gave negative results. Antibiotic therapy (with amoxicillin/clavulanic acid) and daily pleural lavages with saline solution were started, although the patient refused any more-aggressive surgical procedures. The drain was removed 8 days later and the patient was discharged with a C-reactive protein level of $4 \mathrm{mg} \cdot \mathrm{L}^{-1}$ while antibiotics were continued for 3 weeks.

The patient was then not traceable for the next 2 years until they spontaneously presented to an outpatient clinic with a chest radiograph (figure 1d) identical to the very first radiograph (figure 1a). On this occasion the chest radiogram was performed as a preoperative control prior to inguinal hernia repair. The radiological findings shown in figure $1 \mathrm{a}$ and figure $1 \mathrm{~d}$ illustrate well the chronic evolution of a tuberculous pleurisy. At the age of 76 (time of last follow-up), the patient was asymptomatic and in good condition, and no other pulmonary complications were reported.

We think that this case is an interesting example of recurrent tuberculous infection due to reactivation of TB in a patient who was previously "cured". A serious infection by M. Tuberculosis was initially controlled by an adequate anti-TB therapy including streptomycin, rifampin and isoniazid as stated above. It seems likely that the antibiotics were able to decrease the total bacterial count to a minimum but were not able to completely eradicate the infection, which could justify the presence of a chronic pleural fluid with diffuse calcifications. A sort of immunological equilibrium was then reached by the patient and this was maintained until an unexpected superinfection occurred. We would like to hear Dr Petruccioli's opinion about this and whether it could be of any interest and value to screen the patient by tuberculin skin test, interferon- $\gamma$ release assay, or by any other method they describe in their paper.

Our patient presented residual pleural thickening (RPT) due to chronic tuberculous pleurisy. Tuberculous pleurisy is the second most common form of extra-pulmonary tuberculosis, the first being lymphatic

@ERSpublications

Tuberculous pleurisy and subsequent pleural thickening could be associated with recurrent tuberculous infection http://ow.ly/BLGK30aJjvI

Cite this article as: Ampollini L, Bobbio A, Ventura L, et al. Long-lasting tuberculous pleurisy. Eur Respir J 2017; 49: 1602472 [https://doi.org/10.1183/13993003.02472-2016]. 

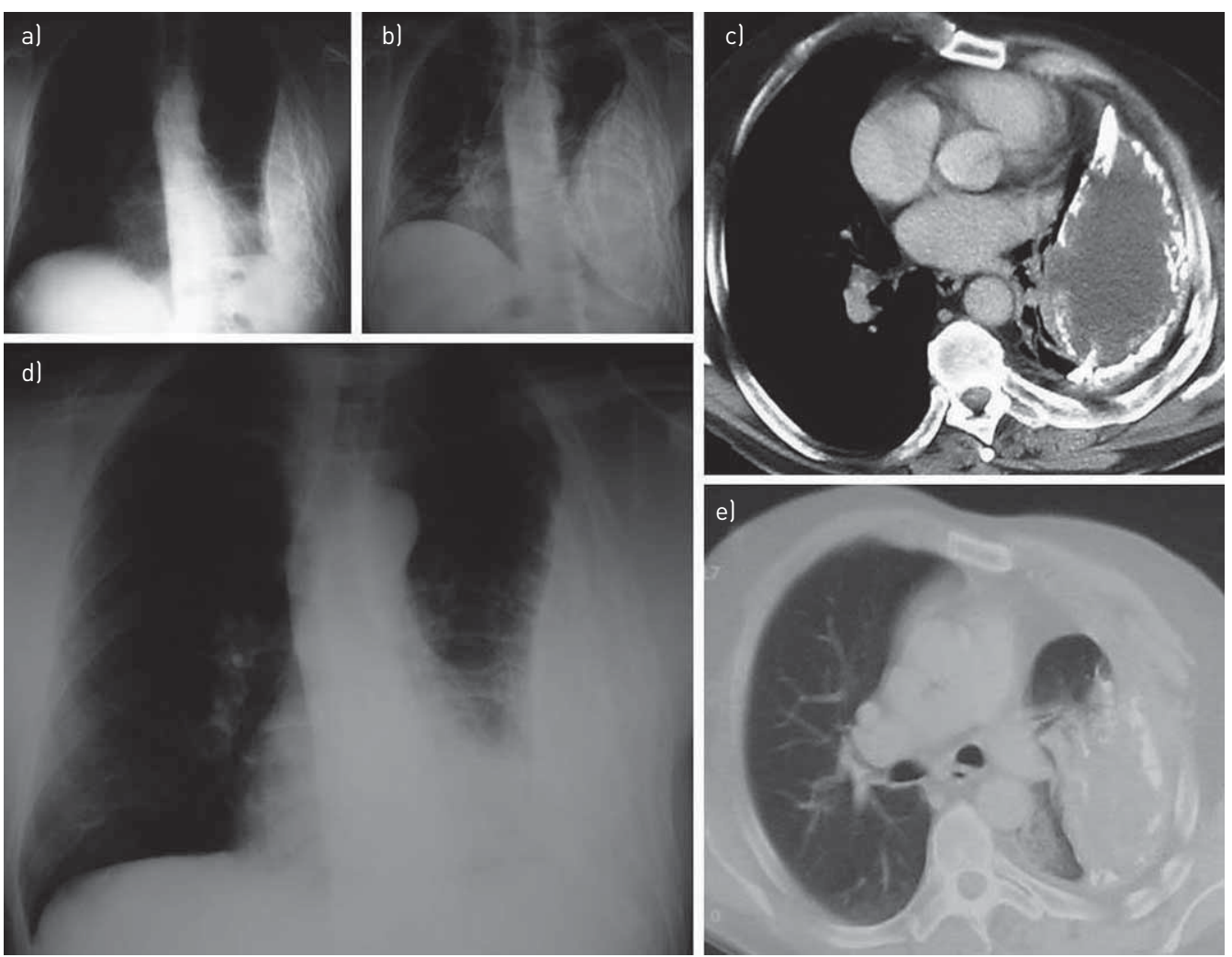

FIGURE 1 Recurrent tuberculous infection in a patient with a long-lasting history of tuberculous pleurisy and secondary residual pleural thickening. The constituent figure parts show a) 46 years old: left chronic pleural effusion likely due to previous tuberculous pleurisy. b) 65 years old: clearly visible enlargement of the pleural collection. c) 65 years old (mediastinal window): chest computed tomography (CT) image confirming the presence of a calcifying pleurisy causing a contralateral mediastinal shift and lung atelectasis. d) 67 years old: left chronic pleural effusion where the radiological appearance is identical to the chest radiogram performed some 20 years previously (figure 1a). e) 65 years old (parenchymal window): chest CT image confirming the presence of a calcifying pleurisy causing a contralateral mediastinal shift and lung atelectasis.

tuberculosis [2-4]. A definitive diagnosis of tuberculous pleurisy usually requires a mycobacterial culture of pleural fluid or a pleural biopsy. The diagnostic yield for a pleural effusion culture was found to range from 12 to $70 \%$ [2, 3]. In 2012, RUAN et al. [2] published a retrospective cohort study showing an effusion culture diagnostic yield of $63 \%$ that increased to $79 \%$ in combination with sputum cultures. They also observed that the lymphocyte percentage of pleural fluid was negatively associated with the probability of a positive effusion culture [2]. This may reflect our own findings, in which the pleural lymphocyte percentage was high and the culture for $M$. tuberculosis was negative. It has been shown that the drainage of pleural effusion, even in the early stages, does not prevent the development of RPT [3]. LAI et al. [5] have reported a randomised study comparing the efficacy of standard anti-TB treatment combined with pigtail drainage to standard anti-TB treatment only. They found that the addition of pigtail drainage was not clinically relevant and did not reduce the level of RPT. Our patient presented an evident RPT over a period of years, despite successful initial anti-TB treatment and numerous subsequent incidences of pleural drainage.

Luca Ampollini ${ }^{1}$, Antonio Bobbio ${ }^{2}$, Luigi Ventura ${ }^{1}$, Claudia Schianchi ${ }^{3}$, Paolo Carbognani ${ }^{1}$ and Michele Rusca ${ }^{1}$ ${ }^{1}$ Thoracic Surgery, Surgical Unit, Dept of Medicine and Surgery, University Hospital of Parma, Parma, Italy. ${ }^{2}$ Dept of Thoracic Surgery, Hôpital Cochin, Assistance publique Hôpitaux de Paris, Paris, France. ${ }^{3}$ Unit of Infectious Diseases and Hepatology, University Hospital of Parma, Parma, Italy.

Correspondence: L. Ampollini, Thoracic Surgery, Surgical Unit, Dept of Medicine and Surgery, University Hospital of Parma, Via Gramsci 14, 43126 Parma, Italy. E-mail: luca.ampollini@unipr.it

Received: Dec 162016 | Accepted after revision: Jan 232017

Conflict of interest: None declared.

Acknowledgements: We would like to thank Petruccioli, Elisa (National Institute for Infectious Diseases "L. Spallanzani", Rome, Italy) and co-workers for their comprehensive work on different tools for predicting the development of active TB from latent TB infection. 


\section{References}

1 Petruccioli E, Scriba T Petrone L, et al. Correlates of tuberculosis risk: predictive biomarkers for progression to active tuberculosis. Eur Respir J 2016; 48: 1751-1763.

2 Ruan SY, Chuang YC, Wang JY, et al. Revisiting tuberculous pleurisy: pleural fluid characteristics and diagnostic yield of mycobacterial culture in an endemic area. Thorax 2012; 67: 822-827.

3 Losi M, Bossink A, Codecasa L, et al. Use of a T-cell interferon-gamma release assay for the diagnosis of tuberculous pleurisy. Eur Respir J 2007; 30: 1173-1179.

4 Porcel JM. Advances in the diagnosis of tuberculous pleuritis. Ann Transl Med 2016; 4: 282.

5 Lai YF, Chao TY, Wang YH, et al. Pigtail drainage in the treatment of tuberculous pleural effusions: a randomised study. Thorax 2003; 58: 149-152.

\section{From the authors:}

We read with interest the correspondence from L. Ampollini and colleagues, in which they report the case of an immunocompetent patient who originally presented with pulmonary and extra-pulmonary tuberculosis (TB) at 33 years of age and which was thought to have persisted over many years. However, based on the clinical story described, we doubt that the patient had reactivation of TB at 65 years of age, when he presented with chest pain and dyspnoea and a computerised tomography (CT)-scan showed a calcifying pleurisy leading to a contralateral mediastinal shift and lung atelectasis. Mycobacterium tuberculosis was not isolated from the pleural effusion. Rather, a positive culture of Streptococcus was obtained and the patient was treated successfully with an anti-streptococcal therapy that was not TB-specific (amoxicillin/clavulanic acid for 3 weeks), with no sign of a TB relapse at follow up 2 years later. Therefore, it seems likely that L. Ampollini and colleagues are describing a case of bacterial pleuritis occurring in a patient with previous TB and calcified sequaele.

Evaluating the case, the clinicians correctly posed the question of reactivation of TB and performed bronchoscopy and microbiological evaluation of the pleural fluid. As a supporting assay, the Quantiferon-TB (Gold-in-Tube) test was also requested. Unfortunately, both of these laboratory tests have suboptimal sensitivity for the diagnosis of TB effusion $[1,2]$; however, they are often performed initially because of their relatively low invasiveness. An additional option might have been the performance of interferon- $\gamma$ release assay and molecular tests on the pleural fluid that may have increased the diagnostic accuracy [2,3]. Following the negative laboratory tests for TB, the clinicians could have performed a pleural biopsy, which is the recommended investigation for confirmation of pleural TB [4], allowing histological evaluation in addition to microbiological and molecular tests.

L. Ampollini and colleagues question whether the biomarkers described as predictive for progression to active TB, the so-called "correlates of risk (COR) for TB progression" [5] may be useful in such clinical cases. In our review, we state that COR may predict progression from $\mathrm{TB}$ infection to active disease; however, the current COR tests are all blood-based and developed for microbiologically-confirmed pulmonary TB. To our knowledge, these assays have never been tested or validated in extra-pulmonary TB. It is therefore difficult to comment on the application of such biomarkers to the reported case without speculating on their utility in extra-pulmonary TB. Nevertheless, the described COR may have diagnostic potential, as described in ZAK et al. [6]. Although biomarkers which are of specific interest to L. Ampollini and colleagues (for the prediction of TB reactivation) have not been described, this issue may yet be addressed by further transcript data mining [6] or by studying specific CD4/CD8 T-cell responses [7, 8] or variation in CD27 expression $[8,9]$. Recently, positron emission tomography and CT-imaging, along with the demonstration of $M$. tuberculosis mRNA in clinical samples, have been described as promising research tools [10] to evaluate incomplete TB "cures". Therefore, we agree with L. Ampollini and colleagues, the scientific community needs to strengthen and focus research to find better tools to predict and monitor TB-treatment outcomes and potential for reactivation.

@ERSpublications

It is necessary to find better tools to predict and monitor TB treatment outcomes and potential for reactivation http://ow.ly/CBxr30aLdCE

Cite this article as: Petruccioli E, Scriba TJ, Petrone L, et al. Long-lasting tuberculous pleurisy. Eur Respir J 2017; 49: 1700356 [https://doi.org/10.1183/13993003.00356-2017]. 
Elisa Petruccioli ${ }^{1}$, Thomas J. Scriba ${ }^{2,3}$, Linda Petrone ${ }^{1}$, Mark Hatherill, ${ }^{2,3}$, Daniela M. Cirillo ${ }^{4}$, Simone A. Joosten ${ }^{5}$, Luigi R. Codecasa ${ }^{6}$, Tom H. Ottenhoff ${ }^{5}$, Claudia M. Denkinger ${ }^{7}$ and Delia Goletti ${ }^{1}$

${ }^{1}$ Dept of Epidemiology and Preclinical Research, Translational Research Unit, National Institute for Infectious Diseases "L. Spallanzani", Rome, Italy. ${ }^{2}$ South African Tuberculosis Vaccine Initiative, Institute of Infectious Disease and Molecular Medicine, Cape Town, South Africa. ${ }^{3}$ Division of Immunology, Dept of Pathology, University of Cape Town, Cape Town, South Africa. ${ }^{4}$ Emerging Bacterial Pathogens Unit, Division of Immunology and Infectious Diseases, San Raffaele Scientific Institute, HSR, Milan, Italy. ${ }^{5}$ Leiden University Medical Center, Leiden, The Netherlands. ${ }^{6}$ Istituto Villa Marelli, ASST Grande Ospedale Metropolitano Niguarda, Milan, Italy. ${ }^{7}$ Tuberculosis and Hepatitis Programme, Foundation for Innovative New Diagnostics (FIND), Geneva, Switzerland.

Correspondence: D. Goletti, Translational Research Unit, National Institute for Infectious Diseases "L. Spallanzani", Via Portuense 292, 00149, Rome, Italy. E-mail: delia.goletti@inmi.it

Received: Feb 202017 | Accepted after revision: Feb 242017

Conflict of interest: Disclosures can be found alongside this article at erj.ersjournals.com

\section{References}

Porcel JM. Advances in the diagnosis of tuberculous pleuritis. Ann Transl Med 2016; 4: 282.

2 Pang CS, Shen YC, Tian PW, et al. Accuracy of the interferon-gamma release assay for the diagnosis of tuberculous pleurisy: an updated meta-analysis. PeerJ 2015; 3: e951.

3 Trajman A, da Silva Santos Kleiz de Oliveira EF, Bastos ML, et al. Accuracy of polimerase chain reaction for the diagnosis of pleural tuberculosis. Respir Med 2014; 108: 918-923.

4 Bibby AC, Maskell NA. Pleural biopsies in undiagnosed pleural effusions; Abrams vs image-guided vs thoracoscopic biopsies. Curr Opin Pulm Med 2016; 22: 392-398.

5 Petruccioli E, Scriba TJ, Petrone L, et al. Correlates of tuberculosis risk: predictive biomarkers for progression to active tuberculosis. Eur Respir J 2016; 48: 1751-1763.

6 Zak DE, Penn-Nicholson A, Scriba TJ, et al. ACS and GC6-74 cohort study groups. A blood RNA signature for tuberculosis disease risk: a prospective cohort study. Lancet 2016; 387: 2312-2322.

7 Day CL, Abrahams DA, Lerumo L, et al. Functional capacity of Mycobacterium tuberculosis-specific T cell responses in humans is associated with mycobacterial load. J Immunol 2011; 187: 2222-2232.

8 Chiacchio T, Petruccioli E, Vanini V, et al. Polyfunctional T-cells and effector memory phenotype are associated with active TB in HIV-infected patients. J Infect 2014; 69: 533-545.

9 Petruccioli E, Navarra A, Petrone L, et al. Use of several immunological markers to model the probability of active tuberculosis. Diagn Microbiol Infect Dis 2016; 86: 169-171.

10 Malherbe ST, Shenai S, Ronacher K, et al. Persisting positron emission tomography lesion activity and Mycobacterium tuberculosis mRNA after tuberculosis cure. Nat Med 2016; 22: 1094-1100.

Copyright @ERS 2017 